\title{
Universal isocontours for dissipative Kerr solitons
}

\author{
Xinbal Li, ${ }^{1,2, \dagger}$ Boqiang Shen,,${ }^{1, \dagger}$ Heming Wang, ${ }^{1, \dagger}$ KI Youl Yang, ${ }^{1, \dagger}$ (i) Xu Yl, ${ }^{1}$ QI-Fan Yang, ${ }^{1}$ \\ Zhiping Z HOU, ${ }^{2}$ and KerRy VAhala ${ }^{1, *}$ \\ ${ }^{1}$ T. J. Watson Laboratory of Applied Physics, California Institute of Technology, Pasadena, California 91125, USA \\ ${ }^{2}$ State Key Laboratory of Advanced Optical Communication Systems and Networks, School of Electronics Engineering and Computer Science, \\ Peking University, Beijing 100871, China \\ *Corresponding author: vahala@caltech.edu
}

Received 19 February 2018; revised 24 April 2018; accepted 26 April 2018; posted 26 April 2018 (Doc. ID 323414); published 23 May 2018

\begin{abstract}
Dissipative Kerr solitons can be generated within an existence region defined on a space of normalized pumping power versus cavity-pump detuning frequency. The contours of constant soliton power and constant pulse width in this region are studied through measurement and simulation. Such isocontours impart structure to the existence region and improve understanding of soliton locking and stabilization methods. As part of the study, dimensionless, closed-form expressions for soliton power and pulse width are developed (including Raman contributions). They provide isocontours in close agreement with those from the full simulation, and, as universal expressions, can simplify the estimation of soliton properties across a wide range of systems. (๑) 2018 Optical Society of America
\end{abstract}

OCIS codes: (190.5530) Pulse propagation and temporal solitons; (140.3945) Microcavities; (190.0190) Nonlinear optics.

https://doi.org/10.1364/OL.43.002567

Provided under the terms of the OSA Open Access Publishing Agreement

Temporal optical solitons resulting from the balance of dispersion with the Kerr nonlinearity have long been studied in optical fiber systems $[1,2]$. In addition to their many remarkable properties, these nonlinear waves are important in modelocking [3], continuum generation [4], and were once considered as a means to send information over great distances [5,6]. Recently, a new type of dissipative temporal soliton [7] was observed in optical fiber resonators [8]. These coherently driven cavity solitons (CSs) were previously considered a theoretical possibility [9], and related soliton phenomena including breather solitons and Raman interactions have also been reported in this system [10-12]. While leveraging the Kerr effect to balance dispersion, this soliton also regenerates using Kerr-induced parametric amplification [13]. Their recent demonstration in microcavity systems [14-20] has made possible highly stable frequency microcombs [21,22]. Referred to as dissipative Kerr solitons (DKs) in the microcavity system, soliton phenomena including the Raman self-shift [23-25], optical Cherenkov radiation [16,25-28], multisoliton systems [29-31], and the cogeneration of new types of solitons [32] have been reported. Moreover, the compact soliton microcomb devices are being studied for systems-on-a-chip applications such as dual-comb spectroscopy
[33,34], precision distance measurement [35,36], optical communications [37], and optical frequency synthesis [38].

Regions of stability and existence are well known in driven soliton systems [39]. These properties of DKs and CSs have been studied using the Lugiato-Lefever (LL) equation $[9,40]$ in a space of normalized pumping power and cavity-pump frequency detuning [14,41-43]. In analogy with thermodynamic phase diagrams, this soliton existence diagram also contains other regions of existence including those for breather solitons as well as more complex dynamical phenomena [44-46]. Figure 1 is a typical diagram showing only the stable soliton region. In thermodynamic phase diagrams another useful construct is the isocontour for processes performed with a state variable held constant (e.g., isochors and isotherms) [47]. These contours not only provide a way to understand processes within the framework of the phase diagram but impart structure to the phase diagram that improves intuition of thermodynamical processes. In this Letter, contours of constant soliton power and constant pulse width are measured and compared to theory. Closed-form expressions for normalized power and pulsewidth that include the Raman process are also developed.

The normalized LL equation is shown below as Eq. (1) [14]. The slowly-varying field envelope $\psi$ is defined such that $|\psi|^{2}=$ $(2 g / \kappa) N$ where $N$ is photon number, $\kappa$ is the cavity mode power damping rate, and $g=\hbar \omega_{\mathrm{c}}^{2} c n_{2} /\left(n^{2} V_{0}\right)$ is the Kerr coefficient with material refractive index $n$, Kerr nonlinear index $n_{2}$, optical mode volume $V_{0}$, cavity resonant frequency $\omega_{c}$, Planck's constant $\hbar$, and speed of light $c . \tau \equiv \kappa t / 2$ and $\theta=\phi \sqrt{\kappa / 2 D_{2}}$ are the normalized time and cavity polar coordinate $(\phi)$ where $D_{2}$ is the second-order dispersion parameter [14,15]. $f^{2} \equiv P / P_{\text {th }}$ is the ratio of the input pump power and parametric threshold power $[13,15]$, and $\zeta \equiv\left(\omega_{\mathrm{c}}-\omega_{\mathrm{p}}\right)(2 / \kappa)$ is the normalized frequency detuning between cavity resonant frequency $\omega_{c}$ and pump frequency $\omega_{p} \cdot \gamma \equiv D_{1} \tau_{\mathrm{R}} \sqrt{\kappa / 2 D_{2}}$ is the normalized Raman coefficient where $\tau_{\mathrm{R}}$ is the material Raman constant [24] and $D_{1} / 2 \pi$ is the cavity free-spectral range $[14,15]$. Eq. (1) is as follows:

$$
\frac{\partial \psi}{\partial \tau}=j \frac{1}{2} \frac{\partial^{2} \psi}{\partial \theta^{2}}+j|\psi|^{2} \psi-(1+j \zeta) \psi+j \gamma \frac{\partial|\psi|^{2}}{\partial \theta} \psi+f .
$$

Isopower contours found by solving Eq. (1) are shown in Fig. 1 as red contours. The analysis is performed for a 


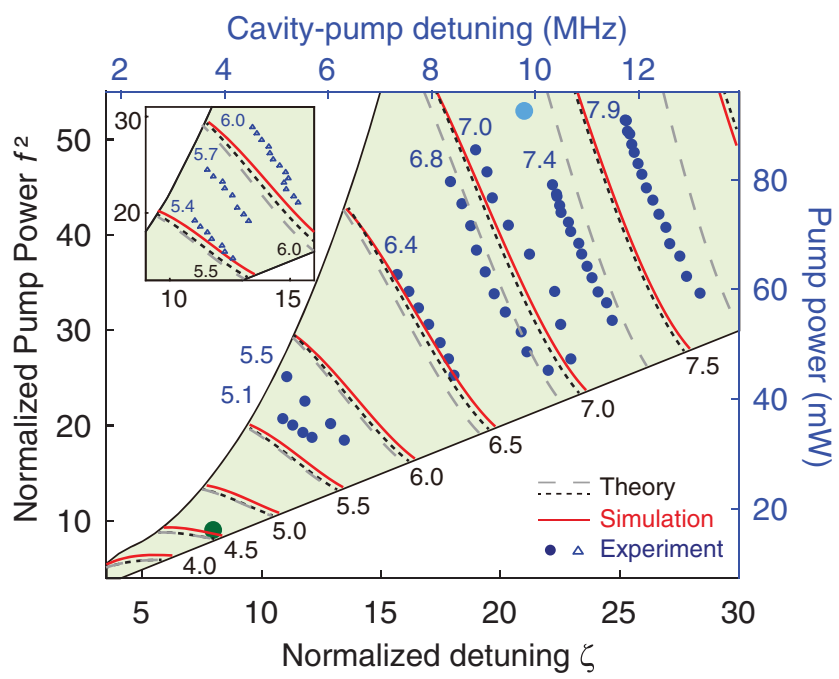

Fig. 1. Dissipative Kerr soliton phase diagram and isopower contours. The phase diagram features normalized pump power $f^{2}$ along the vertical axis and normalized detuning $\zeta$ along the horizontal axis. The green region contains stable soliton states. Black-dotted lines (gray-dashed lines) are isopower contours using Eq. (4) with Raman term (w/o Raman). $p$ is incremented from 4.0 to 8.0 in steps of 0.5 . Red lines are simulated isopower contours using Eq. (1). Blue dots give the measured soliton isopower contours at the soliton powers 93, 99.5, 117.5, 125, 129, 136, and $145 \mu \mathrm{W}$ (left to right), which correspond to $p$ values of $5.1,5.5,6.4,6.8,7.0,7.4$, and 7.9. For these measurements $Q=197$ million $(\kappa / 2 \pi=0.98 \mathrm{MHz})$, $\kappa_{E} / \kappa=0.26$, and $\gamma=2.1 \times 10^{-3}$. Large green and blue data points correspond to spectra in Fig. 2(b). The inset shows the measured isopower contours using another similar device, with soliton powers of 299,320 , and $335 \mu \mathrm{W}$ (left to right), which correspond to $p$ values of 5.4, 5.7, and 6.0. For these measurements $Q=115$ million $(\kappa / 2 \pi=1.69 \mathrm{MHz}), \kappa_{E} / \kappa=0.39$, and $\gamma=2.8 \times 10^{-3}$.

high- $Q$ silica resonator, and parameters used in the calculation are provided below and in the Fig. 1 caption. Numerical simulation is based on propagating the LL equation from an initial soliton seed until steady state is achieved [24].

The following simplified analytical solution for the soliton is also used to study soliton behavior [14]: $\psi=A+$ $B \operatorname{sech}\left(\theta / \tau_{\theta}\right) e^{j \phi_{0}}$, where $A$ is the soliton background field, $B$ is the amplitude, $\phi_{0}$ is the soliton phase, and $\tau_{\theta} \equiv \tau_{s} / \tau_{0}$ is the normalized soliton pulse width $\left(\tau_{s}\right.$ is the physical pulse width and $\left.\tau_{0} \equiv \sqrt{2 D_{2} /\left(\kappa D_{1}^{2}\right)}\right)$. By Fourier transform the soliton spectrum in optical frequency $\nu$ varies as $\operatorname{sech}\left(\nu / \nu_{s}\right)$ where $\nu_{\mathrm{s}} \tau_{\mathrm{s}}=\pi^{-2}$. Approximate expressions giving the Raman-free dependence of amplitude and pulse width on detuning and pump power have been developed $[14,48]$. By including highorder corrections and Raman corrections, improved expressions result as well as an expression for soliton average power,

$$
\begin{gathered}
B(\zeta, f, \gamma) \approx \sqrt{2 \zeta}\left(1+\frac{5}{8} \sqrt{\frac{\pi^{2} f^{2}-8 \zeta}{2 \zeta^{3}}}\right)\left(1-\frac{64}{225} \gamma^{2} \zeta^{3}\right), \\
\tau_{\theta}(\zeta, f, \gamma) \approx \frac{1}{\sqrt{2 \zeta}}\left(1-\frac{1}{4} \sqrt{\frac{\pi^{2} f^{2}-8 \zeta}{2 \zeta^{3}}}\right)\left(1+\frac{64}{225} \gamma^{2} \zeta^{3}\right),
\end{gathered}
$$

$$
p(\zeta, f, \gamma) \approx \sqrt{2 \zeta}\left(1+\sqrt{\frac{\pi^{2} f^{2}-8 \zeta}{2 \zeta^{3}}}\right)\left(1-\frac{64}{225} \gamma^{2} \zeta^{3}\right),
$$

where $p \equiv P_{\text {sol }} / P_{0}$ is the normalized time-averaged soliton power $P_{\text {sol }}$ and $P_{0} \equiv\left(\kappa_{E} \hbar \omega_{c} / \pi g\right) \sqrt{\kappa D_{2} / 2}$ with $\kappa_{E}$ the optical loss rate from waveguide-resonator coupling [15]. As an aside, the requirement of the square root to be real in these expressions $\left(\zeta<\pi^{2} f^{2} / 8\right)$ gives the approximate upper bound of detuning for soliton existence in the phase diagram [14,39,49]. Dotted lines in Fig. 1 are the isopower contours using Eq. (4) (equivalently $p(\zeta, f, \gamma)=$ Constant for $\left.\gamma=2.1 \times 10^{-3}\right)$, and they are in excellent agreement with the simulation contours. Raman contributions become especially important at larger detuning values where the soliton spectrum increases in width [23,24]. To illustrate this point, the dashed curves in Fig. 1 result by using Eq. (4) except with $\gamma=0$.

The experimental setup is shown in Fig. 2(a). The resonator was an ultrahigh- $Q$ silica wedge resonator having a diameter of approximately $3 \mathrm{~mm}$ (free spectral range $D_{1} / 2 \pi=21.9 \mathrm{GHz}$ ). Further details on its fabrication are described in Ref. [50].

(a)
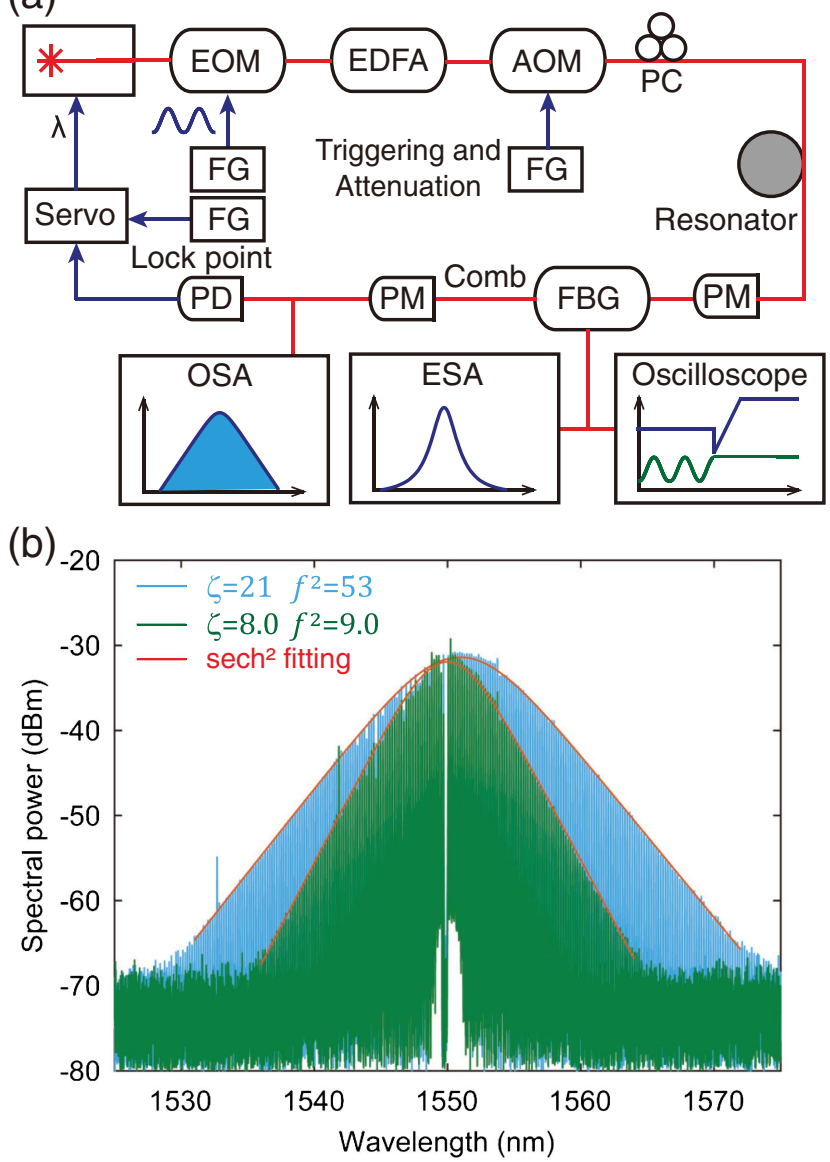

Fig. 2. Measurement system and low power operation. (a) Measurement setup. EOM, electro-optical phase modulator; PC, polarization controller; PM, inline power meter; PD, photodetector; FG, function generator. (b) Soliton spectra at normalized detuning and pumping power $\left(\zeta=21, f^{2}=53\right)$ (blue) and $(\zeta=8.0$, $f^{2}=9.0$ ) (green). The corresponding phase diagram locations are marked in Fig. 1. Red curve: squared hyperbolic-secant fitting. 
The measurement used the $\mathrm{TE}_{1}$ mode family pumped at $1550 \mathrm{~nm}$ and the second-order dispersion was measured to be $D_{2} / 2 \pi=12.1 \mathrm{kHz}$ at $1550 \mathrm{~nm}$ by a method reported elsewhere [15]. The mode area was calculated to be $A_{\text {eff }}=40 \mu \mathrm{m}^{2}$, and the silica Raman constant $\tau_{\mathrm{R}}=2.4 \mathrm{fs}$ was also used [24]; this is valid when the soliton spectral width is below $13 \mathrm{THz}$ [51]. Finally, the resonator used in this measurement featured minimal avoided mode crossings and dispersive waves. Their presence would interfere with the ideal power dependence predicted by the LL equation. To measure pump detuning, weak phase-modulation of the pump light and detection of converted amplitude modulation sidebands were performed $[43,52]$. In this method, pump light reflected by the fiber Bragg grating (FBG) contains the modulation information and is analyzed by an electrical spectrum analyzer (ESA) to retrieve the detuning frequency. The soliton spectrum transmitted past the FBG is sent to a detector and optical spectrum analyzer (OSA) for analysis. To determine soliton power the FBG filtered line was manually reinserted.

Triggering and locking of single soliton states used the soliton average power to servo control the pump laser frequency [53]. Because this soliton locking method maintains a constant soliton power it provides a convenient way to map out the isopower contours. Specifically, as opposed to varying $\left(\zeta, f^{2}\right)$ in the phase diagram and monitoring soliton power, the isopower measurement proceeded by varying only the pumping power with the soliton locked at constant output power. The servo control then compensates for these variations by adjusting the pumping frequency. The corresponding detuning was then recorded as described above. Pump power was varied using a combination of an acousto-optical modulator (AOM) and erbium-doped fiber amplifier (EDFA). Upon completion of an isopower contour, the soliton power setpoint was adjusted and the measurement repeated. The measured isopower data points are shown in the main panel of Fig. 1. Each measurement proceeded until it was no longer possible to reliably lock the soliton state. There is overall good agreement between measurement and theory. Errors are largest at lowest detuning values; however, even here they are relatively small $(\sim 10 \%)$. The ability to measure the contours over such large ranges and their good agreement with theory and simulations showcase the systems robustness and quality. As an additional test, a second loading condition was also measured. The inset to Fig. 1 shows this data, which are in reasonable agreement with the simulation and Eq. (4). Measured soliton powers have experienced a $\sim 1.2 \mathrm{~dB}$ insertion loss between the resonator and the detector. It is also noted that breather solitons could be stably locked near the upper boundary in Fig. 1. However, the region was small. As a result, breathers were not studied in this work.

Stable generation of solitons at small detuning is of practical importance for low pumping power operation of the soliton system. To this end, the green data point $\left(\zeta=8.0, f^{2}=9.0\right)$ in Fig. 1 shows both the lowest detuning and the lowest power soliton state observed in this study. The corresponding unnormalized quantities are $4.2 \mathrm{MHz}$ and $10.8 \mathrm{~mW}$. This is, to the authors' knowledge, the lowest operating power reported for any soliton microcomb platform. Making this result equally important is that the repetition rate is detectable $(21.9 \mathrm{GHz})$ requiring large mode volume and hence higher pumping power levels compared to, for example, terahertz-rate microcombs.
Corresponding soliton spectra are presented in Fig. 2(b). The result was achieved by both the use of a high-quality-factor resonator sample as well as the improved understanding gained through these measurements of the stability regional boundaries [53]. For comparison, a soliton spectrum produced at $\left(\zeta=21, f^{2}=53\right)$ is also shown in Fig. 2(b). These values are plotted as the light blue data point in Fig. 1 and correspond to unnormalized quantities $11.3 \mathrm{MHz}$ and $63.5 \mathrm{~mW}$. The cavity loading condition for these two spectra is: loaded $Q=182$ million and $\kappa_{E} / \kappa=0.44$.

In parallel with the isopower data point collection, the soliton pulse width was also measured by fitting of the optical spectral envelope [15]. Then the data set $\left(\zeta, f^{2}, \tau_{\theta}\right)$ was linearly interpolated to determine isocontours of pulse width (blue contours in Fig. 3). It was not possible to interpolate isopulsewidth contours at lower detuning values where there are fewer isopower data points. For comparison, simulated pulse width (red) and the analytical expression, Eq. (3) (dotted black), are plotted. The interpolated pulse width isocontours are less accurate than the directly measured power contours but nonetheless show reasonable agreement between the data and theory. Overall, the pulse width contours are more weakly dependent upon normalized pumping power (i.e., more vertical) compared to the soliton power contours.

In summary, contours of constant power and constant pulse width have been measured for dissipative Kerr solitons. Measurements were found to be in good agreement with the LL equation numerical model augmented by Raman interactions. There was also good agreement with the predictions of closed-form expressions that include the Raman interaction. Compared to the large-detuning approximation that predicts that soliton power depends only upon resonator-pump detuning (i.e., vertical isopower contours), it is found that soliton power depends both upon pumping power and detuning. The resulting tilt of isopower contours at low detuning suggests

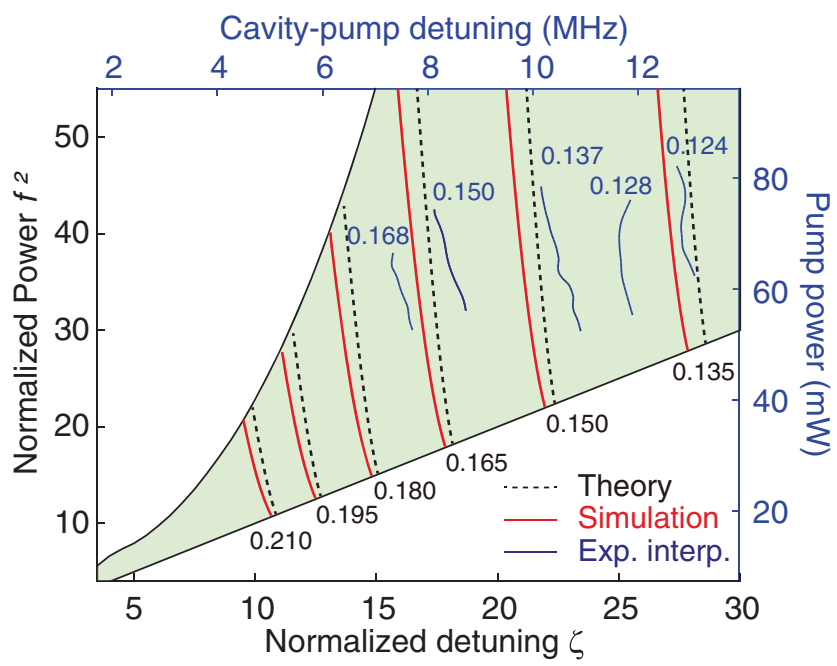

Fig. 3. Isocontours of soliton pulse width. The device is unchanged from the Fig. 1 main panel. Red solid lines (black-dotted lines) are simulated [Eq. (3) theory] isocontours of normalized pulse width $\tau_{\theta}$ ranging from 0.21 to 0.135 (equidistant steps of 0.015 ). Blue solid lines are the linear interpolation from measurement of isocontours at $190,170,155,145$, and $140 \mathrm{fs}$, which correspond to $\tau_{\theta}: 0.168,0.150$, $0.137,0.128$, and 0.124 . 
that soliton locking by servo control of pumping power could potentially be an option for low-detuning ranges just as servo control of pump frequency is used at larger detuning ranges. Stable soliton operation for pump powers as low as $10.8 \mathrm{~mW}$ was also demonstrated in this work. These measurements provide structure to the phase diagram picture of soliton existence. The universal nature of the closed-form expressions should make them suitable for use in other CS and DK soliton platforms. Future work could consider incorporating higher-order dispersion into the analysis to include, for example, the impact of phenomena such as dispersive waves.

Funding. Defense Advanced Research Projects Agency (DARPA) (HR0011-16-C-0118); Kavli Nanoscience Institute.

Acknowledgment. The authors thank Chengying Bao, Yu-Hung Lai, and Myoung-Gyun Suh for helpful discussions. $\mathrm{X}$. Li appreciates the financial support from China Scholarship Council (CSC).

†These authors contributed equally to this work.

\section{REFERENCES}

1. Y. S. Kivshar and G. Agrawal, Optical Solitons: From Fibers to Photonic Crystals (Academic, 2003).

2. G. P. Agrawal, Nonlinear Fiber Optics (Academic, 2007).

3. S. Cundiff, Dissipative Solitons (Springer, 2005), pp. 183-206.

4. J. M. Dudley, G. Genty, and S. Coen, Rev. Mod. Phys. 78, 1135 (2006).

5. A. Hasegawa and Y. Kodama, Proc. IEEE 69, 1145 (1981).

6. H. A. Haus and W. S. Wong, Rev. Mod. Phys. 68, 423 (1996).

7. N. Akhmediev and A. Ankiewicz, Dissipative Solitons: From Optics to Biology and Medicine (Springer, 2008).

8. F. Leo, S. Coen, P. Kockaert, S.-P. Gorza, P. Emplit, and M. Haelterman, Nat. Photonics 4, 471 (2010).

9. S. Wabnitz, Opt. Lett. 18, 601 (1993).

10. J. K. Jang, M. Erkintalo, S. G. Murdoch, and S. Coen, Opt. Lett. 39, 5503 (2014).

11. M. Anderson, F. Leo, S. Coen, M. Erkintalo, and S. G. Murdoch, Optica 3, 1071 (2016).

12. Y. Wang, M. Anderson, S. Coen, S. G. Murdoch, and M. Erkintalo, Phys. Rev. Lett. 120, 053902 (2018).

13. T. Kippenberg, S. Spillane, and K. Vahala, Phys. Rev. Lett. 93, 083904 (2004).

14. T. Herr, V. Brasch, J. Jost, C. Wang, N. Kondratiev, M. Gorodetsky, and T. Kippenberg, Nat. Photonics 8, 145 (2014).

15. X. Yi, Q.-F. Yang, K. Y. Yang, M.-G. Suh, and K. Vahala, Optica 2, 1078 (2015)

16. V. Brasch, M. Geiselmann, T. Herr, G. Lihachev, M. Pfeiffer, M. Gorodetsky, and T. Kippenberg, Science 351, 357 (2016).

17. C. Joshi, J. K. Jang, K. Luke, X. Ji, S. A. Miller, A. Klenner, Y. Okawachi, M. Lipson, and A. L. Gaeta, Opt. Lett. 41, 2565 (2016).

18. P.-H. Wang, J. A. Jaramillo-Villegas, Y. Xuan, X. Xue, C. Bao, D. E. Leaird, M. Qi, and A. M. Weiner, Opt. Express 24, 10890 (2016).

19. M. Yu, Y. Okawachi, A. G. Griffith, M. Lipson, and A. L. Gaeta, Optica 3, 854 (2016).

20. Q. Li, T. C. Briles, D. A. Westly, T. E. Drake, J. R. Stone, B. R. llic, S. A. Diddams, S. B. Papp, and K. Srinivasan, Optica 4, 193 (2017).

21. P. Del'Haye, A. Schliesser, O. Arcizet, T. Wilken, R. Holzwarth, and T. Kippenberg, Nature 450, 1214 (2007).

22. T. J. Kippenberg, R. Holzwarth, and S. Diddams, Science 332, 555 (2011).
23. M. Karpov, H. Guo, A. Kordts, V. Brasch, M. H. Pfeiffer, M. Zervas, M. Geiselmann, and T. J. Kippenberg, Phys. Rev. Lett. 116, 103902 (2016).

24. X. Yi, Q.-F. Yang, K. Y. Yang, and K. Vahala, Opt. Lett. 41, 3419 (2016).

25. X. Yi, Q.-F. Yang, X. Zhang, K. Y. Yang, X. Li, and K. Vahala, Nat. Commun. 8, 14869 (2016).

26. N. Akhmediev and M. Karlsson, Phys. Rev. A 51, 2602 (1995).

27. C. Bao, Y. Xuan, D. E. Leaird, S. Wabnitz, M. Qi, and A. M. Weiner, Optica 4, 1011 (2017).

28. A. Cherenkov, V. Lobanov, and M. Gorodetsky, Phys. Rev. A 95, 033810 (2017).

29. Q.-F. Yang, X. Yi, K. Yang, and K. Vahala, Nat. Photonics 11, 560 (2017).

30. C. Joshi, A. Klenner, Y. Okawachi, M. Yu, K. Luke, X. Ji, M. Lipson, and A. L. Gaeta, "Counter-rotating cavity solitons in a silicon nitride microresonator," arXiv: 1711.04849 (2017).

31. D. C. Cole, E. S. Lamb, P. Del'Haye, S. A. Diddams, and S. B. Papp, Nat. Photonics 11, 671 (2017).

32. Q.-F. Yang, X. Yi, K. Y. Yang, and K. Vahala, Nat. Phys. 13, 53 (2017).

33. M.-G. Suh, Q.-F. Yang, K. Y. Yang, X. Yi, and K. Vahala, Science 354 , 600 (2016).

34. A. Dutt, C. S. Joshi, X. Ji, J. Cardenas, Y. Okawachi, A. L. Gaeta, and M. Lipson, Conference on Lasers and Electro-Optics (CLEO): Science and Innovations (Optical Society of America, 2017), paper STh3L.2.

35. M.-G. Suh and K. J. Vahala, Science 359, 884 (2018).

36. P. Trocha, M. Karpov, D. Ganin, M. H. P. Pfeiffer, A. Kordts, S. Wolf, J. Krockenberger, P. Marin-Palomo, C. Weimann, S. Randel, W. Freude, T. J. Kippenberg, and C. Koos, Science 359, 887 (2018).

37. P. Marin-Palomo, J. N. Kemal, M. Karpov, A. Kordts, J. Pfeifle, M. H. P. Pfeiffer, P. Trocha, S. Wolf, V. Brasch, M. H. Anderson, R Rosenberger, K. Vijayan, W. Freude, T. J. Kippenberg, and C. Koos, Nature 546, 274 (2017)

38. D. T. Spencer, T. Drake, T. C. Briles, J. Stone, L. C. Sinclair, C. Fredrick, Q. Li, D. Westly, B. R. llic, A. Bluestone, N. Volet, T. Komljenovic, L. Chang, S. H. Lee, D. Y. Oh, M. G. Suh, K. Y. Yang, M. H. P. Pfeiffer, T. J. Kippenberg, E. Norberg, L. Theogarajan, K. Vahala, N. R. Newbury, K. Srinivasan, J. E. Bowers, S. A. Diddams, and S. B. Papp, Nature 557, 81 (2018).

39. I. Barashenkov and Y. S. Smirnov, Phys. Rev. E 54, 5707 (1996).

40. L. A. Lugiato and R. Lefever, Phys. Rev. Lett. 58, 2209 (1987).

41. F. Leo, L. Gelens, P. Emplit, M. Haelterman, and S. Coen, Opt. Express 21, 9180 (2013)

42. C. Godey, I. V. Balakireva, A. Coillet, and Y. K. Chembo, Phys. Rev. A 89, 063814 (2014).

43. H. Guo, M. Karpov, E. Lucas, A. Kordts, M. Pfeiffer, V. Brasch, G. Lihachev, V. Lobanov, M. Gorodetsky, and T. Kippenberg, Nat Phys. 13, 94 (2017).

44. C. Bao, J. A. Jaramillo-Villegas, Y. Xuan, D. E. Leaird, M. Qi, and A. M. Weiner, Phys. Rev. Lett. 117, 163901 (2016).

45. E. Lucas, M. Karpov, H. Guo, M. Gorodetsky, and T. Kippenberg, Nat. Commun. 8, 736 (2017)

46. M. Yu, J. K. Jang, Y. Okawachi, A. G. Griffith, K. Luke, S. A. Miller, X. Ji, M. Lipson, and A. L. Gaeta, Nat. Commun. 8, 736 (2017).

47. R. E. Sonntag, C. Borgnakke, G. J. Van Wylen, and S. Van Wyk, Fundamentals of Thermodynamics (Wiley, 2003).

48. H. Guo, M. Karpov, E. Lucas, A. Kordts, M. Pfeiffer, V. Brasch, G. Lihachev, V. Lobanov, M. Gorodetsky, and T. Kippenberg, Nat. Phys. 13, 94 (2016).

49. S. Coen and M. Erkintalo, Opt. Lett. 38, 1790 (2013).

50. H. Lee, T. Chen, J. Li, K. Y. Yang, S. Jeon, O. Painter, and K. J. Vahala, Nat. Photonics 6, 369 (2012).

51. M. Erkintalo, G. Genty, B. Wetzel, and J. M. Dudley, Opt. Express 18 25449 (2010)

52. E. Lucas, H. Guo, J. D. Jost, M. Karpov, and T. J. Kippenberg, Phys. Rev. A 95, 043822 (2017).

53. X. Yi, Q.-F. Yang, K. Y. Yang, and K. Vahala, Opt. Lett. 41, 2037 (2016). 